\title{
Thermo-Resistive Platinum Thin Film Hydrogen Gas Sensor Fabricated by MEMS Techniques
}

\author{
Daisuke Yamazaki Member (Graduate School of IPS, Waseda University) \\ Lin Zhang Student Member (Graduate School of IPS, Waseda University) \\ Joanna Pawlat Non-member (Graduate School of IPS, Waseda University) \\ Toshitsugu Ueda Member (Graduate School of IPS, Waseda University)
}

Keywords : MEMS, platinum thin film, hydrogen gas sensor, lift-off process

In the recent years, the global environment and energy problems have gained considerable attention. The hydrogen fuel begins to be used widely as the next generation energy source. The hydrogen fuel technology is clean and environmental friendly. However, hydrogen is a dangerous gas that explodes easily. It is necessary to develop the high sensitivity, high reliability, cheap hydrogen gas sensor, which will assure safety and will be able to work under the system requirements.

In this study, we developed thermo-resistive platinum(Pt) thin film sensor, which was produced using Micro Electro Mechanical Systems (MEMS) fabrication techniques. The sensor design incorporated resistor elements that would facilitate the temperature-resistance characteristics and mechanisms of Pt based thin film thermo-resistors. The sensor was fabricated with lift-off technique that allowed the dual sensing surface area. Furthermore, the dual sensing surfaces ensured faster response to hydrogen gas. Sensor had an unique structure formed by the removal of the substrate under the thin film, that highly improved the detection of the resistance change in the Pt film. As the result, influence of the substrate on the sensor's work was small, and the response speed was good. Fig. 1 shows the structure of $\mathrm{Pt}$ thin film hydrogen gas sensor.

Fig.2 shows the sensor's characteristics for various hydrogen concentrations. The operating temperatures were $60^{\circ} \mathrm{C}, 80^{\circ} \mathrm{C}$, $100^{\circ} \mathrm{C}, 120^{\circ} \mathrm{C}$ and the concentration of hydrogen gas in air ranged from $2 \%$ to $9 \%$. At the same hydrogen concentration, the output voltage of the sensor was higher when the self-current heating temperature of sensor's Pt thin film increased. Thus, the sensor sensitivity improved with the thin film's temperature rising.

Additionally, the differential Pt thin film hydrogen gas sensor

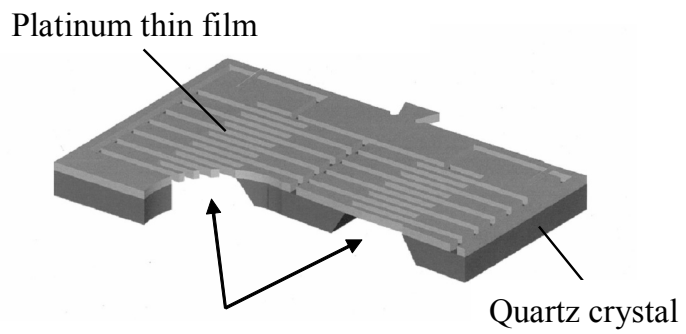

Quartz crystal parts removed by etching

Fig. 1. Structure of Pt thin film hydrogen gas sensor was developed. This gas sensor was able to compensate the changes in the external environment such as temperature, moisture, etc. Sensor's body incorporated two structures: one with the Pt thin film only, and the other where Pt thin film was completely covered with the thin layer of $\mathrm{Cr}$, therefore, catalyst reaction did not occur there. Both structures of the sensor were manufactured in the same way. Fig.3 shows the photograph of differential Pt thin film hydrogen gas sensor.

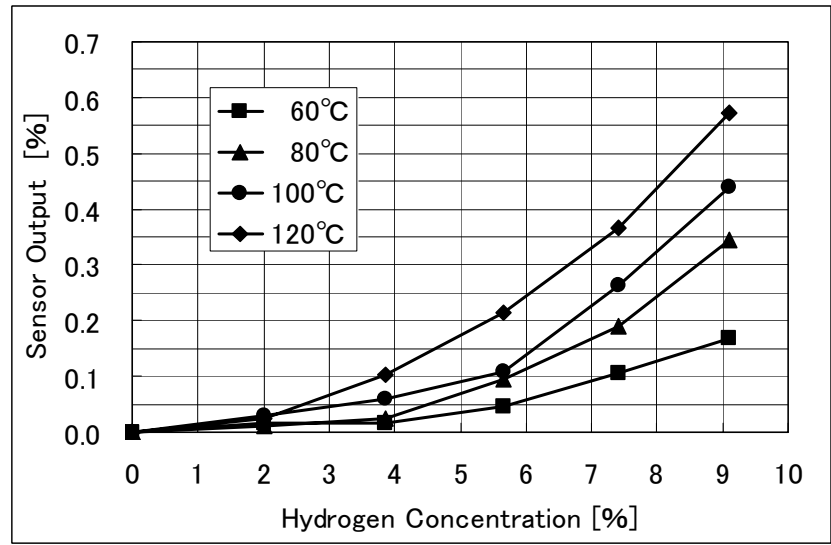

Fig. 2. Sensor's characteristics for various hydrogen concentrations

(Operating temperatures: $60^{\circ} \mathrm{C}-120^{\circ} \mathrm{C}$ )

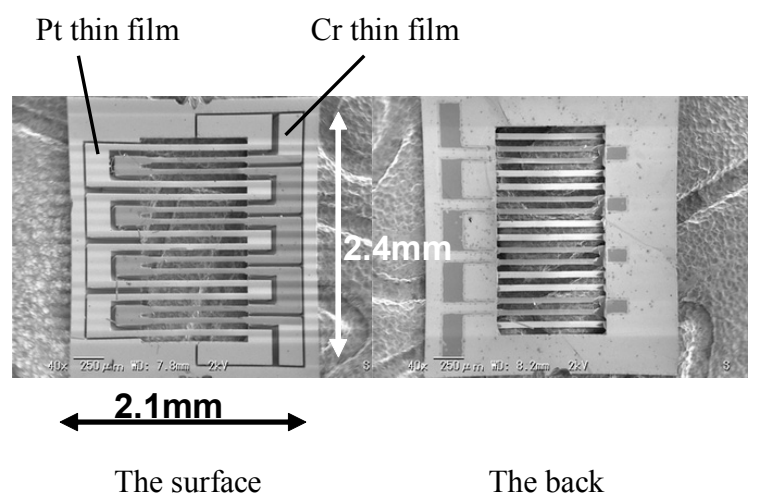

Fig. 3. Photograph of differential $\mathrm{Pt}$ thin film hydrogen gas sensor 


\title{
Thermo-Resistive Platinum Thin Film Hydrogen Gas Sensor Fabricated by MEMS Techniques
}

\author{
Daisuke Yamazaki* Member \\ Lin Zhang* Student Member \\ Joanna Pawlat* Non-member \\ Toshitsugu Ueda* Member
}

\begin{abstract}
Thermo-resistive platinum(Pt) thin film sensor was produced using Micro Electro Mechanical Systems (MEMS) fabrication techniques. The sensor design incorporated resistor elements that would facilitate the temperature-resistance characteristics and mechanisms of Pt based thin film thermo-resistors. The sensor was fabricated with lift-off process that allowed the dual sensing surface area. Furthermore, the dual sensing surfaces ensured faster response to hydrogen gas. To increase the catalytic reaction comparing with at the room temperature, the sensor was heated to a specified temperature by applying the current to a Pt thin film. The catalytic reaction took place when the heated sensor came into the contact with hydrogen. Additionally, the differential Pt thin film hydrogen gas sensor was also developed. This gas sensor was able to compensate the changes in the external environment such as temperature, moisture, etc. The fabricated hydrogen sensor detected the concentration of hydrogen gas in air from $2 \%$ to $9 \%$.
\end{abstract}

Keywords : MEMS, platinum thin film, hydrogen gas sensor, lift-off process

\section{Introduction}

In the recent years, the global environment and energy problems have gained considerable attention. The hydrogen fuel begins to be used widely as the next generation energy source. The hydrogen fuel technology is clean and environmental friendly. However, hydrogen is a dangerous gas that explodes easily. It is necessary to develop high sensitive, high reliable, cheap hydrogen gas sensors, which will assure safety and will be able to work under the system requirements. Various hydrogen sensors have been proposed so far including the contact combustion type gas sensor, the semiconductor type gas sensor, the ultrasonic wave type gas sensor ${ }^{(1)}$, and the thermoelectric gas sensor ${ }^{(2)}$. According to the recent studies, the detection of hydrogen concentration for the thermoelectric gas sensor ranged from $0.5 \mathrm{ppm}$ to $5 \%$. Additionally, the Pd-Ni alloy thin film hydrogen sensor of the SAW type reports on $10 \mathrm{ppm}-100 \%$ concentration range. The principle is the change of elasticity characteristic due to adsorption of hydrogen into Pd-Ni alloy film. Still, sensors available on the market require higher precision, faster response time and reliability ${ }^{(3)-(5)}$.

In this paper, thermo-resistive Pt thin film sensor, which was produced using Micro Electro Mechanical Systems (MEMS) fabrication techniques is proposed. The sensor design incorporated resistor elements that would facilitate the temperature-resistance characteristics and mechanisms of $\mathrm{Pt}$ based thin film thermo-resistors. The sensor was fabricated with lift-off technique that allowed the dual sensing surface area. Furthermore, the dual sensing surfaces enabled faster response to hydrogen gas. Sensor had an unique structure formed by the removal of the substrate

* Graduate School of Information, Production and Systems, Waseda University

Wakamatsu-ku, Kitakyushyu, Fukuoka, 808-0135 under the thin film, that highly improved the detection of the resistance change in the Pt film. As the result, influence of the substrate on the sensor's work was small, and the response speed was high. Additionally, the differential Pt thin film hydrogen gas sensor was developed. This gas sensor was able to compensate the changes in the external environment such as temperature, moisture, etc. Sensor's body incorporated two structures: one with the $\mathrm{Pt}$ thin film only, and the other where Pt thin film was completely covered with the thin layer of chromium $(\mathrm{Cr})$, therefore, catalyst reaction did not occur there. The goal of this study was to develop a low cost Pt thin film sensor having high sensitivity, wide sensing range and high reliability.

\section{Methods of hydrogen detection}

2.1 Pt Catalysis It is expected that a reaction speed of the catalyst varies depending on its thickness, surface area, temperature etc. Fick's first law (1) expresses the diffusion flux J of hydrogen in the Pt thin film ${ }^{(6)-(8)}$

$$
J=-\frac{D}{R T}\left(\frac{d p}{d x}\right) \quad\left[\frac{\mathrm{mol}}{\mathrm{m}^{2} s}\right]
$$

where $D$ : Diffusion coefficient $\left[\mathrm{m}^{2} \mathrm{~s}^{-1}\right]$

$R:$ Gas constant (8.3143) $\left[\mathrm{J} \mathrm{mol}^{-1} \mathrm{~K}^{-1}\right]$

$T$ : Absolute temperature $[\mathrm{K}]$

$\frac{d p}{d x}$ : Pressure gradient $\left[\frac{\mathrm{Pa}}{\mathrm{m}}\right]$

Calculation of an actual calorific value of the reactive gas requires the diffusion coefficient of the gas:

$$
\begin{aligned}
& D_{H_{2}}=6.61 \times 10^{-5}\left[\mathrm{~m}^{2} \mathrm{~s}^{-1}\right] \\
& \text { In the condition of } \mathrm{T}=80^{\circ} \mathrm{C} \\
& J_{H_{2}}=0.0345\left[\mathrm{~mol} \mathrm{~m}^{-2} \mathrm{~s}^{-1}\right]
\end{aligned}
$$

Chemical equation (2) describes the reactive thermochemistry of hydrogen and oxygen. The calorific value of $1 \mathrm{~mol}$ of hydrogen is 
$285.8 \mathrm{~kJ}$.

$$
\mathrm{H}_{2}+\frac{1}{2} \mathrm{O}_{2}=\mathrm{H}_{2} \mathrm{O}-285.8 \mathrm{~kJ}
$$

In this case, the calorific value for Pt thin film's unit area can be calculated as follows (3):

$$
0.0345 \frac{\mathrm{mol}}{\mathrm{m}^{2} \mathrm{~s}} \times 285.8 \frac{\mathrm{kJ}}{\mathrm{mol}}=0.986 \frac{\mathrm{W}}{\mathrm{cm}^{2}}
$$

2.2 Thermal-Resistive Sensor The Pt thin film resistance changes due to the temperature variations, according to the expression (4).

$$
\begin{aligned}
& R_{t}=R_{0}\left(1+\alpha T-\beta T^{2}\right) \\
& R_{0}: \text { Resistance at } 0^{\circ} \mathrm{C} \\
& R_{t}: \text { Resistance at } \mathrm{T}^{\circ} \mathrm{C} \\
& \alpha: \text { First order temperature coefficient }\left[{ }^{\circ} \mathrm{C}^{-1}\right] \\
& \beta: \text { Second order temperature coefficient }\left[{ }^{\circ} \mathrm{C}^{-2}\right] \\
& T: \text { Temperature }\left[{ }^{\circ} \mathrm{C}\right]
\end{aligned}
$$

To increase the catalytic reaction, the sensor was heated to a specified temperature by appyling the current to a Pt thin film. The catalytic reaction took place when the heated sensor came into the contact with hydrogen.

\section{Fabrication Process of Pt Thin Film}

Fig.1 shows the structure of Pt thin film hydrogen gas sensor. The diagram of the fabrication process for proposed device is presented in Fig.2. Sensor was produced using various MEMS fabrication techniques. Magnetron sputtering system enabled the deposition of different types of films i.e. $\mathrm{Cr}$, $\mathrm{Au}$, Pt onto the quartz crystal wafer. The Pt is well known as a stable metal, which is etched with difficulty by any of existing enchants. The only easy way to pattern Pt thin film is using lift-off technique. We used two kinds of resists for a new lift-off process to make an accurate $\mathrm{Pt}$ thin film. The quartz crystal substrate was etched by wet etching technique. The experimental procedure can be summarized as follows.

(a) The quartz crystal wafer was cleaned in $96 \%$ sulfuric acid and $30 \%$ hydrogen peroxide solution. $\mathrm{Cr}$ and $\mathrm{Au}$ thin films were sputterd on both side of the quartz crystal wafer.

(b) Lift-off resist and photoresist were spread on both sides of the quartz crystal wafer. Pt patterns were formed during photolithography and eching.

(c) Pt was sputterd on both sides of the quartz crystal wafer.

(d) Pt patterns were formed using lift off techniques.

(e) $\mathrm{Cr}$ and $\mathrm{Au}$ thin films were etched.

(f) The quartz crystal wafer was etched with the metal film as a mask.

(g) Finally, $\mathrm{Cr}$ and $\mathrm{Au}$ thin films were etched.

The important features of designed hydrogen sensor were the following points:

(1) Both sides of the Pt thin film could be used as a sensor because there was no substrate.

(2) Temperature of the catalyst could be easily raised.

\section{Results and Discussion}

4.1 Experimental Apparatus The schematic diagram and the photograph of experimental set up are presented in Fig.3 and Fig.4, respectively. The sensing characteristics of the device were measured using specially designed hydrogen testing equipment. To increase the catalytic reaction, the sensor was heated to the specified temperature by applying the current to the Pt thin film. The temperature of the sensor was controlled by the
Platinum thin film

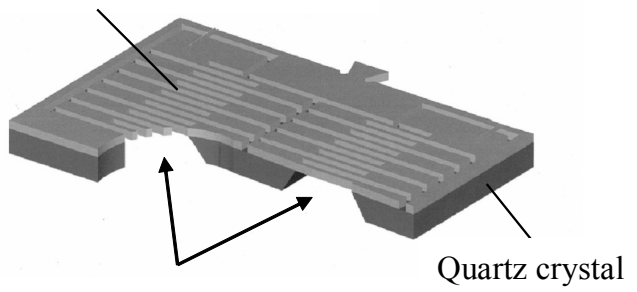

Quartz crystal parts removed by etching

Fig. 1. Structure of $\mathrm{Pt}$ thin film hydrogen gas sensor.

(a)

(b)

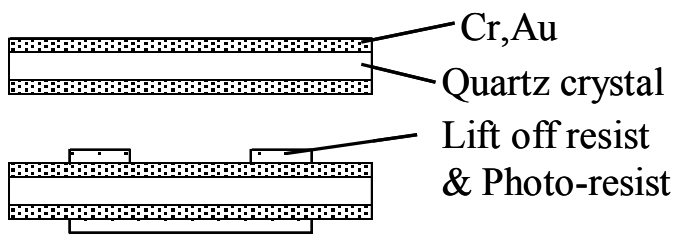

(c)

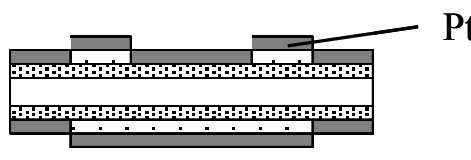

(d)

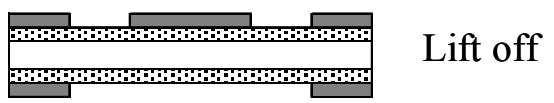

(e)

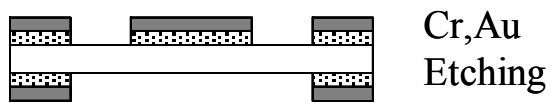

(f)

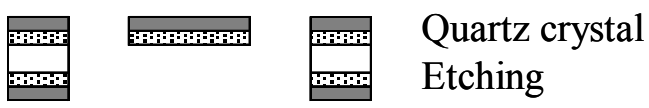

(g)

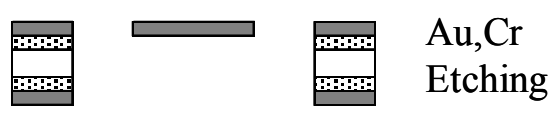

Fig. 2. Fabrication process for Pt thin film sensor.

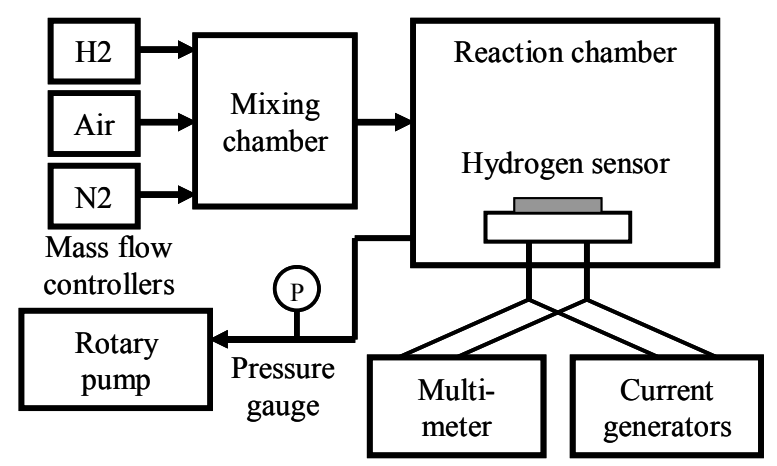

Fig. 3. Schematic diagram of experimental set up.

supplied current value. The concentration of the hydrogen in the chamber was adjusted using the mass flow controllers. The catalytic reaction took place when the heated sensor came into contact with hydrogen. At the same time, there was a change in resistance of the sensor, which was measured by a multi-meter. The gases used in experiment were hydrogen, air, and nitrogen. Pressure in the reaction chamber was monitored using pressure 


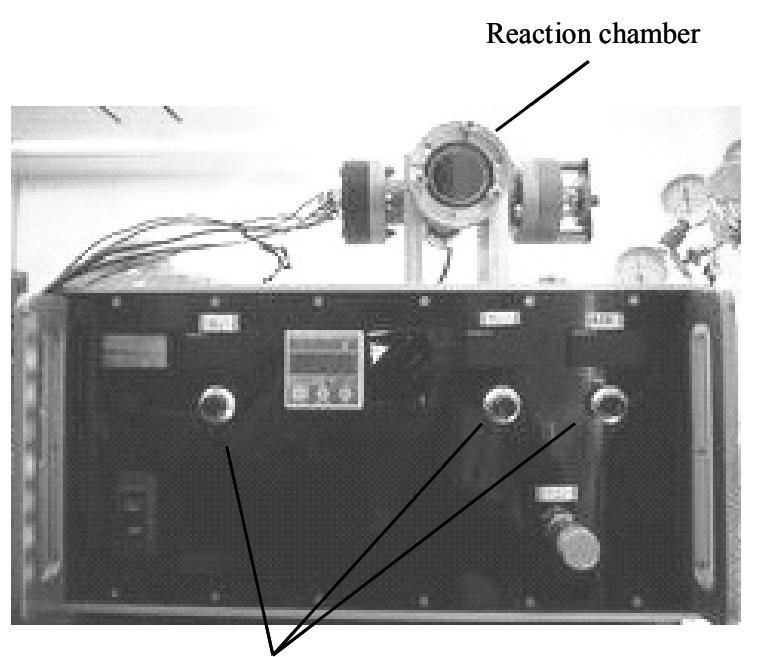

Mass flow controllers

Fig. 4. Photograph of experimental set up.

gauge. The rotary pump assured the accurate concentration of the hydrogen in the reaction chamber during the measurements.

4.2 Experimental Results Fig.5 shows the sensor output as function of hydrogen concentration and sensor temperature. The operating temperatures were $60^{\circ} \mathrm{C}, 80^{\circ} \mathrm{C}, 100^{\circ} \mathrm{C}$, $120^{\circ} \mathrm{C}$ and the concentration of hydrogen gas in air ranged from $2 \%$ to $9 \%$. In the same hydrogen concentration, the output voltage of the sensor was higher when the self-current heating temperature of sensor's Pt thin film increased. Thus, the sensor sensitivity improved with the thin film's temperature rising. Expression (3) after the concentration's converting gives $0.0197 \mathrm{~W} / \mathrm{cm}^{2} / \%$. Experimental result was $0.00491 \mathrm{~W} / \mathrm{cm}^{2} / \%$. The experimental value was four times smaller, but it admits an improvement comparing with the work conducted by the other groups and is still expected to be improved.

The sensor's characteristics for various hydrogen concentrations in dependence on pressure are presented in Fig.6. The operating gas pressures were $100 \mathrm{kPa}, 150 \mathrm{kPa}, 200 \mathrm{kPa}$ and the concentration of hydrogen gas in air ranged from $2 \%$ to $9 \%$. The temperature of the sensor was $80^{\circ} \mathrm{C}$. At the same hydrogen concentration, the output voltage of the sensor was higher when the larger pressures, which enhanced the revitalization of catalyst were applied.

Fig. 7 shows the response characteristic of the sensor in the $10 \%$ hydrogen concentration. The time constant of the sensor was about 50 seconds.

4.3 Differential Hydrogen Sensor The differential Pt thin film hydrogen gas sensor was developed. This gas sensor was able to compensate the changes in the external environment such as temperature, moisture, etc. Sensor's body incorporated two structures: one with the Pt thin film only, and the other where $\mathrm{Pt}$ thin film was completely covered with the thin layer of $\mathrm{Cr}$, therefore, catalyst reaction did not occur there. Both structures of the sensor were manufactured in the same way. Each resistance R can be calculated as follows (5):

$$
R=\rho \frac{l}{w \cdot t} \quad[\Omega]
$$

where $\rho$ : Volume resistivity of material $[\Omega \cdot m]$

$$
\begin{array}{ll}
l & : \text { Length of resistor }[\mathrm{m}] \\
w & : \text { Width of resistor }[\mathrm{m}] \\
t & : \text { Thickness of resistor }[\mathrm{m}]
\end{array}
$$

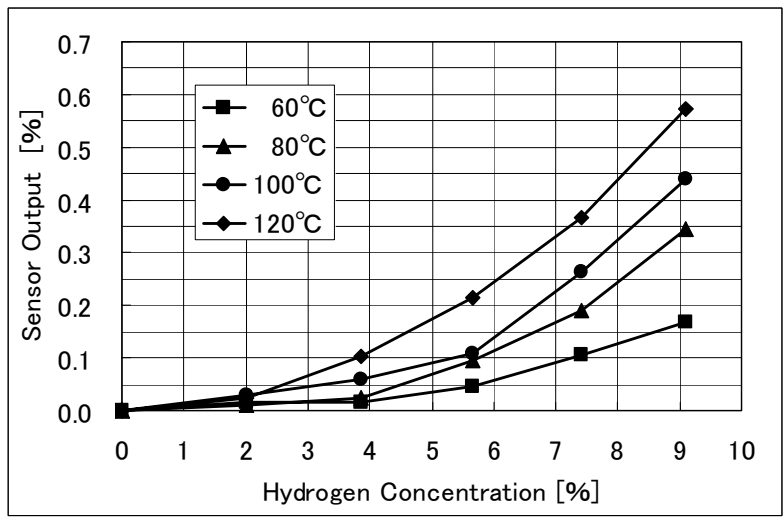

Fig. 5. Sensor's characteristics for various hydrogen concentrations. (Operating temperatures: $60^{\circ} \mathrm{C}-10^{\circ} \mathrm{C}$ )

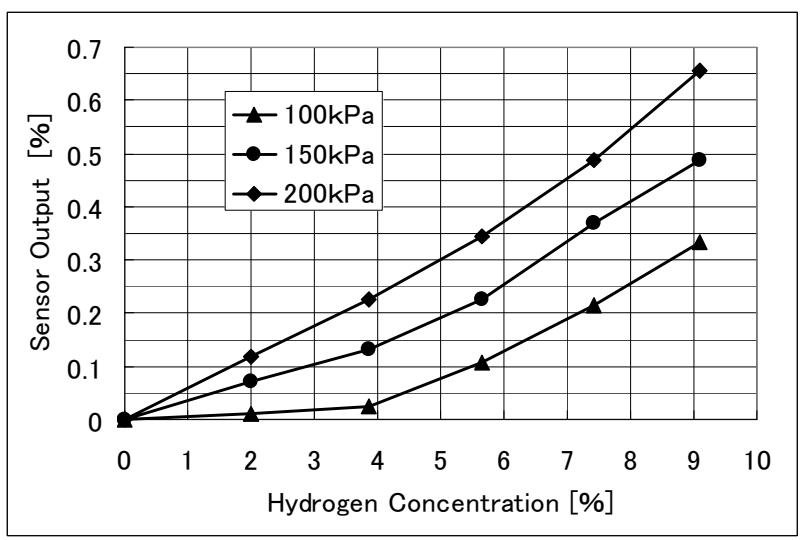

Fig. 6. Sensor's characteristics for various hydrogen concentrations. (Operating pressures: $100 \mathrm{kPa}-200 \mathrm{kPa}$ )

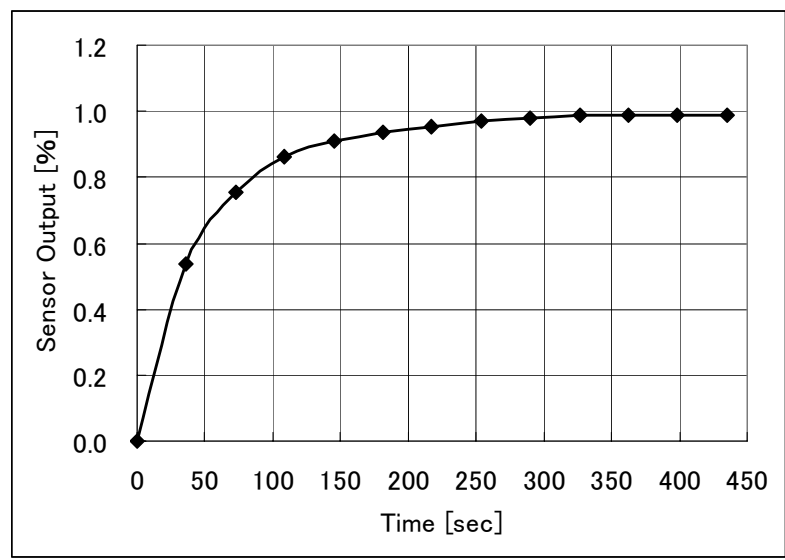

Fig. 7. The response characteristic of the sensor in the $10 \%$ hydrogen concentration. (Operating temperatures: $\left.80^{\circ} \mathrm{C}\right)$

The dimensions of proposed device (length, width, and thickness) were designed in such a way that each resistance was the same. Additionally, the following points could describe the features of the sensor:

(1) The Pt thin film and the $\mathrm{Cr}$ thin film were alternately adjacent because it made the temperature of the sensor the same.

(2) The Pt film and the Cr film were fabricated as the beam structure for insulation.

Fig. 8 shows the mask pattern of a differential Pt thin film 
Pt thin film pattern

Cr thin film pattern

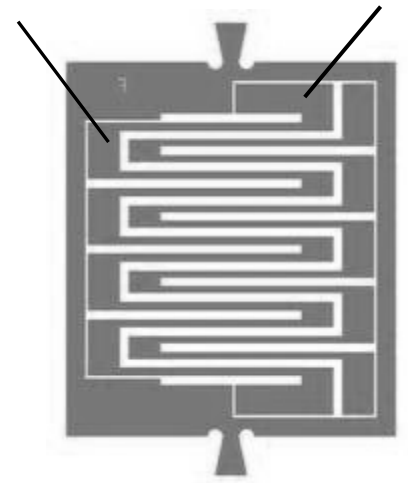

Fig. 8. Mask pattern of differential Pt thin film hydrogen gas sensor.

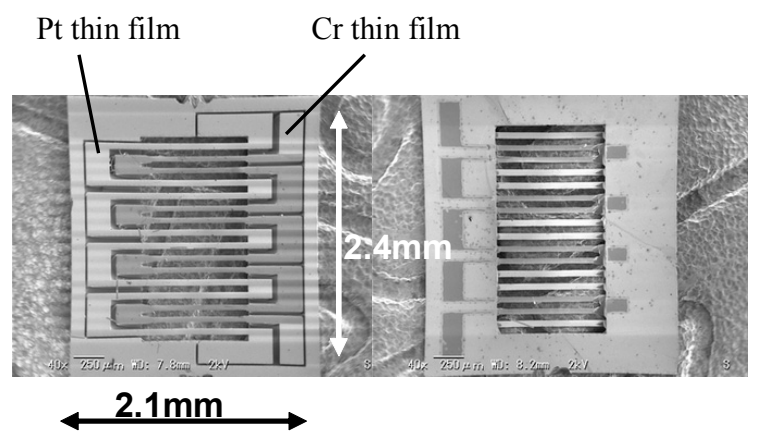

The surface

The back

Fig. 9. Photograph of differential $\mathrm{Pt}$ thin film hydrogen gas sensor.

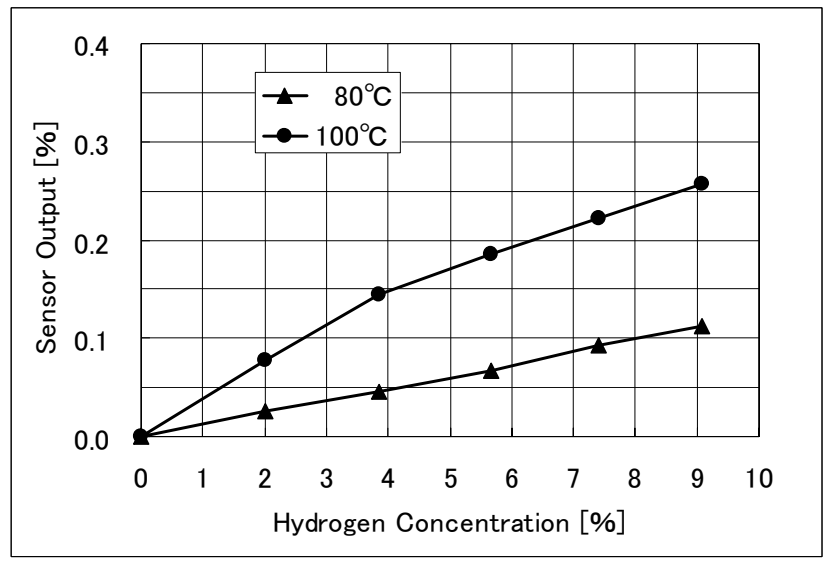

Fig. 10. Sensor's characteristics for various hydrogen concentrations. (Operating temperatures: $80^{\circ} \mathrm{C}-100^{\circ} \mathrm{C}$ )

hydrogen gas sensor.

The photograph of sensor is presented in Fig.9. The size of the device was relatively small: $2.1 \mathrm{~mm} \times 2.4 \mathrm{~mm}$.

Fig.10 depicts the differential sensor's characteristics for various hydrogen concentrations in dependence on temperature. As in the previous case, at the same hydrogen concentration, the output voltage of the sensor was higher when the self-current heating temperature of sensor's Pt thin film increased. It might be assumed that the sensor output was small because the differential
Differential platinum thin film hydrogen gas sensor

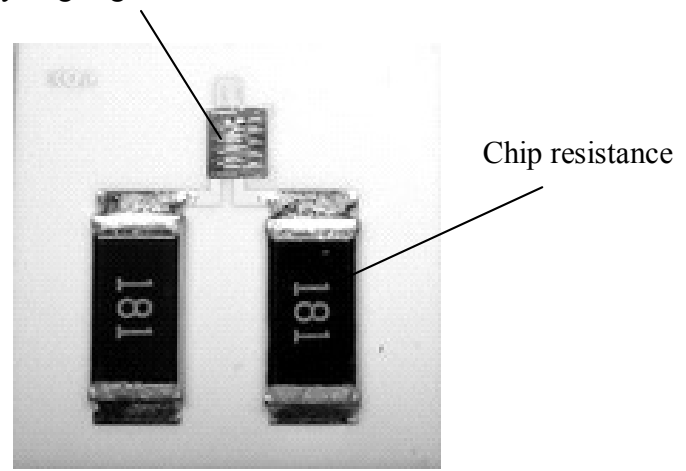

Fig. 11. Photograph of package structure of differential Pt thin film hydrogen gas sensor.

structure was still not complete.

The package structure of differential device is shown in Fig.11. To assure the precision of further tests, the Pt thin film sensor and the chip resistor were bridged.

\section{Conclusions}

In this study, the Pt thin film sensor that was able to use both sides of the Pt film was produced using the MEMS technology.

System that measured the sensor's sensitivity was designed, and the generation of heat in the effect of catalytic reaction with hydrogen was measured.

The influence of temperature and pressure on the sensitivity of the device for various concentrations of hydrogen was clarified.

Additionally, the differential Pt thin film hydrogen gas sensor was developed and its evaluation was performed.

After further improvements a high sensitivity, wide range, high reliability sensing device is expected to be manufactured.

(Manuscript received Aug. 30, 2007, revised Apr. 8, 2008)

\section{References}

(1) K. Yamanaka, S. Ishikawa, N. Nakaso, and N. Takeda : "Ball SAW device for hydrogen gas sensor", Ultrasonics Symposium, IEEE (2003)

(2) M. Nishibori, W. Shin, L. F. Houlet, K. Tajima, T. Itoh, N. Izu, N. Murayama, and I. Matsubara : "New structural design of microthermoelectric sensor for wide range hydrogn detection", Journal of Ceramic Society of Japan, Vol.144, 10, pp.853-856 (2006)

(3) W. Shin, M. Matsumiya, F. Qiu, N. Izu, and N. Murayama "Thermmoelectric gas sensor for detection of high hydrogen concentration", Sensors and Actuators, B97, pp.344-347 (2004)

(4) H. Ikeda, J. Sato, and F. A. Williams : "Surface kinetics for hydrogen-air mixtures on platinum at atmosperic pressure in stagnation flows", Surface science, 326, pp.11-26 (1995)

(5) M. Matsumiya, W. Shin, N. Izu, and N. Murayama : "Nano-structured thin-film Pt catalyst for thermoelectric hydrogen gas sensor", Sensors and Actuators, B93, pp.309-315 (2003)

(6) L. G. Petersson and Ulf Ackelid : "Kinetic studies of diffusion limited gas-surface reactions by spatially resolved gas samling: reaction rates and sticking coefficients in the $\mathrm{H} 2+\mathrm{O} 2$ reaction on $\mathrm{Pt}$ ", Surface science, 269/270, pp.500-505 (1992)

(7) S. J. Gentry and T. A. Jones : "The role of catalysis in solid-states gas sensors", Sensors and Actuators, 10, pp.141-163 (1986)

(8) Ulf Ackelid, J. Fogelbsrg, and L. G. Petersson : "Local gas sampling and surface hydrogen detection in catalysis on planar surfaces", Vacuum, 42, pp.889-895 (1991) 
Daisuke Yamazaki (Member) received his B.E. and M.E. degree in fine

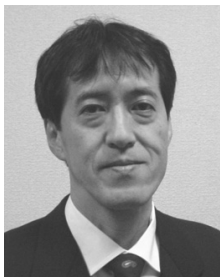

mechanical engineering from Shizuoka University (Japan), in 1980 and 1982, respectively. Since joining Yokogawa Electric Corporation in 1982, he has been engaged in developing sensors for measuring physical and chemical quantities such as temperature, pressure, flow rate. $\mathrm{He}$ is currently working on Ph.D. degree in Graduate School of Information, Production and Systems in Waseda University. Mr. Yamazaki is a member of the Institute of Electrical Engineers of Japan and Society of Instrument and Control Engineers of Japan.

Lin Zhang (Student Member) received the B.Com degree in

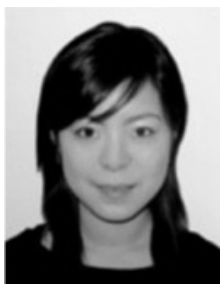
Business and Commercial school from Kyushu International University, Japan, in 2006. She is currently a graduate student in the Graduate School of Information, Production and Systems in Waseda University. She is engaged in research and development of the platinum thin film hydrogen gas sensor.
Joanna Pawlat (Non-member) received her M.E. and M.Sc. degree

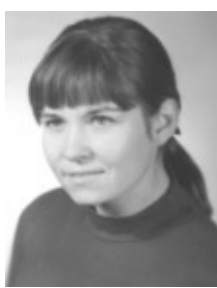
in Sanitary and Building Engineering in 1997 and in Environmental Protection in 1998, from Technical University of Lublin (Poland). She receved D.Sc. degree from Saga University (Japan) on forming phenomena in 2001. She was a post doctor resercher in the Department of Electrical and Electrical Engineering of Chuo University and JSPS reserch fellow in Saga University, cooperating with Nagoya University. Presently, she is an assistant professor in Waseda University working on the gas sensing and foaming phenomena.

Toshitsugu Ueda (Member) was born in 1945. He received his B.E.

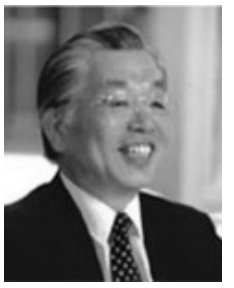
and M.E. degree in electrical engineering from Shinshu University, Nagano, Japan, in 1969 and 1971, respectively. He received Ph.D. degree from Tokyo Institute of Technology in 1988. From 1971 to 2003 , he has been engaged in developing low noise amplifiers, mechanical resonators, micro machining technologies for temperature, pressure and displacement in Yokogawa Electric Corporation. Since 2003 he is a professor of Graduate School of Information, Production and Systems in Waseda University in Fukuoka (Japan), working on sensors and sensor systems. He received Awards from Society of Instrument and Control Engineers of Japan in 1987 and 1994, and Awards form Japan Institute Invention and Innovation in 1985 and 1987. Professor Ueda is a member of the institute of Electrical Engineers of Japan, and Society of Instrument and Control Engineers of Japan. 